University of New Hampshire

University of New Hampshire Scholars' Repository

$11-2008$

\title{
Observations of clustering inside oceanic bubble clouds and the effect on short-range acoustic propagation
}

Thomas C. Weber

University of New Hampshire, Durham, thomas.weber@unh.edu

Follow this and additional works at: https://scholars.unh.edu/ccom

Part of the Oceanography and Atmospheric Sciences and Meteorology Commons

\section{Recommended Citation}

T. C. Weber, 'Observations of clustering inside oceanic bubble clouds and the effect on short-range acoustic propagation', The Journal of the Acoustical Society of America, vol. 124, no. 5, p. 2783, 2008.

This Journal Article is brought to you for free and open access by the Center for Coastal and Ocean Mapping at University of New Hampshire Scholars' Repository. It has been accepted for inclusion in Center for Coastal and Ocean Mapping by an authorized administrator of University of New Hampshire Scholars' Repository. For more information, please contact Scholarly.Communication@unh.edu. 


\title{
Observations of clustering inside oceanic bubble clouds and the effect on short-range acoustic propagation
}

\author{
Thomas C. Weber \\ Center for Coastal and Ocean Mapping, University of New Hampshire, Durham, New Hampshire 03824
}

(Received 11 January 2008; revised 20 June 2008; accepted 27 August 2008)

\begin{abstract}
It has recently been shown [Weber, T. C. et al. (2007). "Acoustic propagation through clustered bubble clouds," IEEE J. Ocean. Eng. 32, 513-523] that gas bubble clustering plays a role in determining the acoustic field characteristics of bubbly fluids. In particular, it has been shown that clustering changes the bubble-induced attenuation as well as the ping-to-ping variability in the acoustic field. The degree to which bubble clustering exists in nature, however, is unknown. This paper describes a method for quantifying bubble clustering using a high frequency $(400 \mathrm{kHz})$ multibeam sonar, and reports on observations of near-surface bubble clustering during a storm (14.6 m/s wind speed) in the Gulf of Maine. The multibeam sonar data are analyzed to estimate the pair correlation function, a measure of bubble clustering. In order to account for clustering in the mean acoustic field, a modification to the effective medium wave number is made. With this modification, the multibeam sonar observations are used to predict the effect of clustering on the attenuation of the mean field for short-range propagation $(1 \mathrm{~m})$ at frequencies between 10 and $350 \mathrm{kHz}$. Results for this specific case show that clustering can cause the attenuation to change by 20\%-80\% over this frequency range. (C) 2008 Acoustical Society of America.
\end{abstract}

[DOI: 10.1121/1.2990707]

PACS number(s): 43.30.Ft [RCG]

Pages: 2783-2792

\section{INTRODUCTION}

It has long been recognized that the propagation of acoustic waves in fluid media is changed in the presence of gas bubbles (Mallock, 1910; Wood, 1930), and that the performance of sonar systems can be adversely affected by increased sound attenuation and changes in sound speed (National Defense Research Committee, 1946). The theories that have been set forth and widely used to describe these changes treat the two-phase bubbly mixtures as a single "effective" medium with characteristics describing the average propagation (Mallock, 1910; Wood, 1930; Foldy, 1945; Van Wijngaarder, 1972; Commander and Prosperetti, 1989; Henyey, 1999). Regardless of the approach used when developing these theories (multiple scattering, the continuum approximation, and the diagram method), it is often assumed that the locations of the bubbles are statistically independent random variables.

Weber et al. (2007) explored the changes in acoustic field statistics in the presence of statistical dependence on the positions of the bubbles, a scenario which is described as bubble clustering after Shaw et al. (2002) and is analogous to the preferential concentrations of particles explored by Eaton and Fessler (1994). Utilizing the multiple scattering approach developed by Foldy (1945) and an artificial model for bubble clustering, it was found that for bubble clouds in which both single and double scattering were important, the presence of bubble clustering changes both the attenuation of the coherent pressure field and the variability of the pressure amplitude (note that the term double scattering is used here to refer to scattering chains that have one interaction with two different bubbles). Examining a simulated population of $100 \mu \mathrm{m}$ bubbles at their resonance frequency, Weber et al.
(2007) found that clustering caused a decrease in the attenuation and an increase in the variability from that which would be expected from a nonclustered bubble cloud.

In general, the bubble-induced attenuation for scattering chains containing up to two bubbles can be calculated by examining the average pressure field observed at some field point $\vec{r}$ due to some distant source of sound as follows:

$$
\begin{aligned}
\langle p(\vec{r})\rangle= & p_{0}(\vec{r})+\int_{V} \int s\left(a_{i}\right) p_{0}\left(\vec{r}_{i}\right) G\left(\vec{r}, \vec{r}_{i}\right) n\left(\vec{r}_{i}, a_{i}\right) d a_{i} d \vec{r}_{i} \\
& +\int_{V} \int_{V} \iint s\left(a_{i 1}\right) s\left(a_{i 2}\right) p_{0}\left(\vec{r}_{i 1}\right) G\left(\vec{r}_{i 1}, \vec{r}_{i 2}\right) G\left(\vec{r}_{,} \vec{r}_{i 2}\right) \\
& \times g\left(\left|\vec{r}_{i 1}-\vec{r}_{i 2}\right|\right) n\left(\vec{r}_{i 1}, a_{i 1}\right) n\left(\vec{r}_{i 2}, a_{i 2}\right) d a_{i 1} d a_{i 2} d \vec{r}_{i} d \vec{r}_{i},
\end{aligned}
$$

which is the sum of the bubble-free pressure $p_{0}$, a single scattering term (the double integral), and a double scattering term (the quadruple integral). Each bubble is assumed to have a complex scattering coefficient $s$, which is a function of the radius $a$. The propagation along each scattering path is described by the free-space Green's function $G$. The positions and sizes of the bubbles within the volume $V$ are treated as random variables; the bubble size distribution $n(\vec{r}, a)$ is proportional to the marginal probability density function describing the position and size of each bubble. In the event of bubble clustering, the positions of two bubbles will not be statistically independent. That is, the term clustering is used here to imply that, given the location of a single bubble, the probability of finding a second bubble nearby is higher than the probability of finding it far away. In Eq. (1), clustering is represented in the double scattering term by the use of the pair correlation function $g$, which is parametrized on the dis- 
tance between the two bubbles in the double scattering chain. As described by Weber et al. (2007), this pair correlation term is used to relate the joint probability density function to the marginal probability density functions for each bubble as follows:

$$
n\left(\vec{r}_{i 1}, \vec{r}_{i 2}\right)=n\left(\vec{r}_{i 1}\right) n\left(\vec{r}_{i 2}\right) g\left(\left|\vec{r}_{i 1}-\vec{r}_{i 2}\right|\right) .
$$

If there is no clustering, then the locations of the bubbles are statistically independent and the pair correlation function $g$ is equal to 1 . When the bubbles are clustered, the pair correlation function will be greater than 1 at short separation distances, but should still be convergent to 1 at large distances where the clustering becomes uncorrelated. It is important to note that for higher orders of scattering, higher order correlation functions are needed: Eqs. (1) and (2) describe scattering chains including up to two bubbles, and hence, the pair correlation function is required; triple scattering would require the three point correlation function, and so on.

A natural extension of the work described by Weber et al. (2007), who utilized an artificial model of bubble clustering, is to quantify the degree of bubble clustering that can be found in either natural (e.g., under oceanic breaking waves) or man-made (e.g., in ship wakes) environments. The source of clustering may be irregularities in the process creating the bubbles (collapsing air cavities for breaking waves or cavitation sites/rates for ship propellers), or turbulence if there is a gradient in the mean spatial distribution of bubbles (as is the case in the ocean, with higher quantities of bubbles at the surface than at depth). Quantifying clustering in bubble clouds is best done by direct measurements of correlation functions, such as the pair correlation function $g$, which are nominally parametrized as a function of the separation distance between the bubbles. It seems reasonable to expect, however, that these correlation functions will change as the dynamics causing the bubble clustering change. For example, in bubble clouds under breaking waves, the correlation functions may change as the wind speed (and therefore wave height) changes, there may be a depth dependency that is related to the dissipation of wave energy, and so on.

This paper describes a method for extracting the pair correlation function from high frequency $(400 \mathrm{kHz})$ multibeam sonar measurements (Sec. II), and presents observations of bubble clustering under oceanic breaking waves in a single wind-wave condition (Sec. III). These observations are taken from a measurement of opportunity when the ship on which the multibeam sonar was mounted was returning to port because of bad weather, traveling through open ocean breaking waves, and the bubble clouds beneath them during a storm with a sustained $14.6 \mathrm{~m} / \mathrm{s}$ wind speed. A modification to the effective medium wave number that accounts for clustering is presented, and a prediction of the attenuation of the mean acoustic field is made for short-range propagation $(1 \mathrm{~m})$ at frequencies between 10 and $350 \mathrm{kHz}$ (Sec. IV).

\section{ESTIMATING THE PAIR CORRELATION FUNCTION}

Historically, multibeam sonars have primarily been used for seafloor mapping (Medwin and Clay, 1988), but more recently have been used for mapping quantities of interest in the water column, including bubbles (Weber et al., 2005), pelagic fishes (Mayer et al., 2002; Gerlotto and Paramo, 2003), marine mammals (Benoit-Bird and Au, 2003), particulates (Jones, 2003), and turbulent microstructure (Trevorrow, 2005). Typically, these sonars are high frequency, narrowband active sonars mounted in a downward or sideward looking mode on a wide range of vessels (e.g., $10 \mathrm{~m}$ launches, icebreakers, and ocean-going research vessels). They most often utilize a Mills cross array configuration (Mills and Little, 1953) with orthogonal transmit and receive arrays that are used to achieve both high spatial resolution (as small as $0.5^{\circ}$ beamwidth) and a large field of view, most typically resulting in a wide fan of beams that interrogate a narrow slice of the water column. Similar to conventional echo sounders, some multibeam sonars, such as the one used in this work, provide the capability to record backscatter from scatterers within the water column for each receive beam.

In some scenarios, the pair correlation function for bubbles, or other scatterers, can be estimated directly from the multibeam sonar backscatter measurements. Consider the backscattered pressures $p_{1}$ and $p_{2}$ observed in two different multibeam sonar resolution cells (or voxels) on a single ping (i.e., for a single configuration of the bubbles) as follows:

$$
\begin{aligned}
& p_{1}(\vec{r})=\sum_{i=N_{1}}^{N_{2}} s\left(a_{i}\right) p_{0}\left(\vec{r}_{i}\right) G\left(\vec{r}, \vec{r}_{i}\right), \\
& p_{2}(\vec{r})=\sum_{i=N_{3}}^{N_{4}} s\left(a_{i}\right) p_{0}\left(\vec{r}_{i}\right) G\left(\vec{r}, \vec{r}_{i}\right),
\end{aligned}
$$

where the scattered pressure observed at the receiving array located at $\vec{r}$ is the sum of the scattered waves from each set of bubbles within the resolution cell (for $N$ bubbles in the cloud, it is assumed that bubbles $N_{1}-N_{2}$ contribute to the backscattered pressure $p_{1}$, and $N_{3}-N_{4}$ contribute to $p_{2}$ ). The incident pressure $p_{0}$ at each bubble is that due to the acoustic field that would exist in the absence of any bubbles, $s\left(a_{i}\right)$ is the scattering coefficient for bubble $i$ with radius $a_{i}$, and the scattered acoustic wave propagates from each bubble, located at $\vec{r}_{i}$ to the receiving array according to the free-space Green's function $G$. There are two important observations to make about Eq. (3): It is assumed that only single scattering is important, and the bubble populations in both resolution cells are assumed to be different. The correlation between the two observations can then be written as

$$
\begin{aligned}
\left\langle p_{1}(\vec{r}) p_{2}(\vec{r})\right\rangle= & \int_{V_{i}} \int_{V_{j}} \iint s\left(a_{i}\right) s\left(a_{j}\right) p_{0}\left(\vec{r}_{i}\right) p_{0}\left(\vec{r}_{j}\right) \\
& \times G\left(\vec{r}, \vec{r}_{i}\right) G\left(\vec{r}, \vec{r}_{j}\right) n\left(a_{i}, a_{j}, \vec{r}_{i}, \vec{r}_{j}\right) d a_{i} d a_{j} d \vec{r}_{i} d \vec{r}_{j},
\end{aligned}
$$

where \langle\rangle indicates an expected moment. The integrals over volumes $V_{i}$ and $V_{j}$ correspond to the two resolution cells, and the bubble size distribution $n$ is proportional to the joint probability density function $\rho$ describing the position and size of each bubble as follows: 


$$
\rho\left(a_{i}, a_{j}, \vec{r}_{i}, \vec{r}_{j}\right)=(1 / N)^{2} n\left(a_{i}, a_{j}, \vec{r}_{i}, \vec{r}_{j}\right) .
$$

Assuming that the bubble sizes are independent of one another and not a function of position, and that the joint probability density function describing the locations of the bubbles at $\vec{r}_{i}$ and $\vec{r}_{j}$ is equal to the product of the pair correlation function $g$ and the squared marginal probability density function (Weber et al., 2007), then Eq. (4) can be written as

$$
\begin{aligned}
\left\langle p_{1}(\vec{r}) p_{2}(\vec{r})\right\rangle= & \int_{V_{i}} \int_{V_{j}} \iint s\left(a_{i}\right) s\left(a_{j}\right) p_{0}\left(\vec{r}_{i}\right) p_{0}\left(\vec{r}_{j}\right) G\left(\vec{r}, \vec{r}_{i}\right) \\
& \times G\left(\vec{r}, \vec{r}_{j}\right) g\left(\left|\vec{r}_{i}-\vec{r}_{j}\right|\right) n\left(a_{i}, \vec{r}_{i}\right) n\left(a_{j}, \vec{r}_{j}\right) d a_{i} d a_{j} d \vec{r}_{i} d \vec{r}_{j} .
\end{aligned}
$$

If it is further assumed that the pair correlation function is independent of the position over the volume integrals (which correspond to the resolution cells of the multibeam sonar), the correlation function can be written as

$$
\begin{aligned}
\left\langle p_{1}(\vec{r}) p_{2}(\vec{r})\right\rangle= & g(r) \int_{V_{i}} \int s\left(a_{i}\right) p_{0}\left(\vec{r}_{i}\right) G\left(\vec{r}, \vec{r}_{i}\right) n\left(a_{i}, \vec{r}_{i}\right) d a_{i} d \vec{r}_{i} \\
& \times \int_{V_{j}} \int s\left(a_{j}\right) p_{0}\left(\vec{r}_{j}\right) G\left(\vec{r}, \vec{r}_{j}\right) n\left(a_{j}, \vec{r}_{j}\right) d a_{j} d \vec{r}_{j} \\
= & g(r)\left\langle p_{1}(\vec{r})\right\rangle\left\langle p_{2}(\vec{r})\right\rangle .
\end{aligned}
$$

Thus, the pair correlation function $g(r)$, where $r$ is the distance separating the two resolution cells located at $\vec{r}_{i}$ and $\vec{r}_{j}$, is

$$
g(r)=\frac{\left\langle p_{1}(\vec{r}) p_{2}(\vec{r})\right\rangle}{\left\langle p_{1}(\vec{r})\right\rangle\left\langle p_{2}(\vec{r})\right\rangle}
$$

Here, $p_{1}$ and $p_{2}$ are proportional to the measurements directly recorded from the multibeam sonar. The constants of proportionality could be found by calibrating the sonar so that the digitized returns from the sonar can be converted exactly into backscattered pressures, but are actually not required here because they would simply cancel out in Eq. (8). Further, the constants of proportionality are not required to be the same for both $p_{1}$ and $p_{2}$ if, for example, the volume of the multibeam resolution cells were different. Thus, any calibration error that might propagate forward into the pair correlation estimate error is canceled out. It is also worth noting that completely uncorrelated backscattered pressures would have a pair correlation of 1 according to the definition given by Eq. (8).

\section{OBSERVATIONS OF THE PAIR CORRELATION FUNCTION}

\section{A. Environmental conditions}

The methodology for estimating the pair correlation function described in Sec. II has been applied to multibeam data collected in the Gulf of Maine approximately $150 \mathrm{~km}$ east of Cape Cod, MA on 5 October 2006 aboard the R/V Hugh R. Sharp. During this time, the R/V Sharp was transiting in a westerly direction at a speed over ground of approximately $4 \mathrm{~m} / \mathrm{s}$, en route to Woods Hole, MA due to the dete-
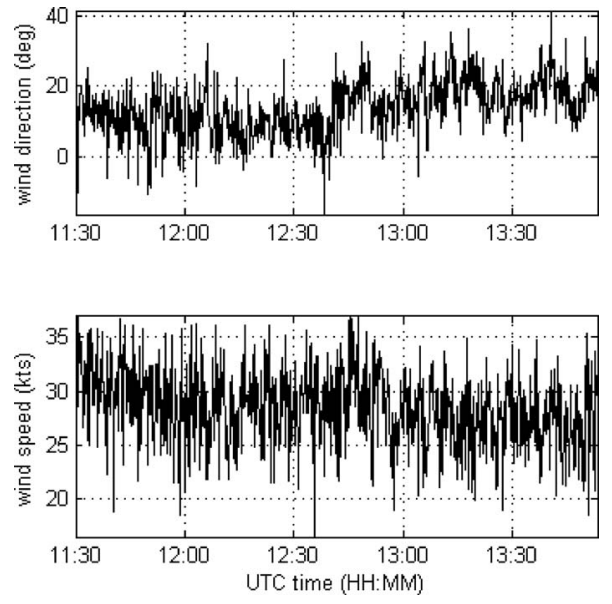

FIG. 1. The wind speed and direction during the multibeam sonar measurements on 5 October 2006.

rioration of weather conditions. Of particular interest are data collected between 11:30 and 13:30 UTC. The average wind speed observed on the ship's mast at a height of $17 \mathrm{~m}$ during this time period was $14.6 \mathrm{~m} / \mathrm{s}$ with a standard deviation of $1.8 \mathrm{~m} / \mathrm{s}$, and the average wind direction was $14^{\circ} \mathrm{T}$ with a standard deviation of $8^{\circ}$ (Fig. 1).

Bubbles created under wind generated breaking waves are a ubiquitous feature of the ocean surface layer $(\mathrm{Wu}$, 1981; Thorpe, 1982; Crawford and Farmer, 1987; Wu, 1998). The surface expression of a wave breaking event is a white cap, and it is estimated that for wind speeds of $10 \mathrm{~m} / \mathrm{s}$, approximately $1 \%$ of the surface will be covered by whitecaps (Monahan and Lu, 1990). Underneath these white caps are bubble clouds with void fractions of gas that have been observed to be as high as $10^{-3}$ (Terrill and Melville, 1997). Some attempts have been made to parametrize the bubble size distributions created in these circumstances as a function of wind speed and depth in the water. The parametrization that will be used here is from Novarini et al. (1998), which is based on the work done by both Monahan and Lu (1990) and Wu (1994).

The evolution of a bubble cloud can be separated into different stages: the first second or so after breaking where the void fraction of air can reach as high as $10^{-1}$, the subsequent few seconds after breaking where the bubble plume is being advected downward by the jet associated with the breaking wave with void fractions as high as $10^{-3}-10^{-4}$, the final stage where the breaking wave energy has largely decayed and the cloud of bubbles is more strongly affected by local currents and processes such as Langmuir circulation, where void fractions are $\mathrm{O}\left(10^{-6}\right)$, and the eventual decay into a background population of bubbles that has a very long lifetime (Monahan and Lu, 1990; Novarini et al., 1998). The bubble clouds associated with a particular breaking wave have been defined as $\alpha-, \beta$-, and $\gamma$-plumes, reflecting the changes in the cloud as it ages to $1 \mathrm{~s}, 3-4 \mathrm{~s}$, and beyond (Monahan and Lu, 1990). Novarini et al. (1998) have parametrized the bubble size distribution and void fraction as a function of wind speed and depth, using a cubic wind speed dependence and an exponential decay in depth for the void 


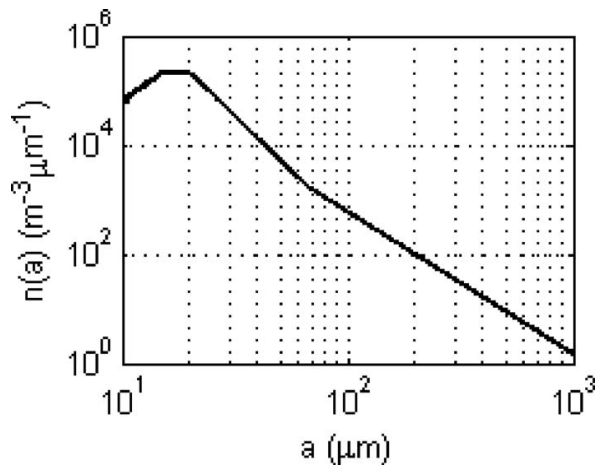

FIG. 2. The bubble size distribution for a $\beta$-plume at a wind speed of $14.6 \mathrm{~m} / \mathrm{s}$ and a depth of $6 \mathrm{~m}$ using the parametrization given by Novarini $e t$ al. (1998).

fraction. Using this parametrization with a wind speed of $14.6 \mathrm{~m} / \mathrm{s}$ and a depth of $6 \mathrm{~m}$ results in a void fraction of $4.5 \times 10^{-6}$ and the bubble size distribution shown in Fig. 2 .

\section{B. Multibeam sonar measurements}

Measurements of bubble clouds during the R/V Sharp's transit to Woods Hole, MA were made with a Reson 7125 SeaBat multibeam sonar. This sonar was mounted at a depth of approximately $4 \mathrm{~m}$, looking downward, in one of the transducer bays in the retractable keel of the R/V Sharp. Gated cw pulses at a frequency of $400 \mathrm{kHz}$ were transmitted from a linear transducer array oriented to generate a beam pattern that is approximately $1^{\circ}$ wide at the half-power points $(-3 \mathrm{~dB})$ in the fore-aft direction and $155^{\circ}$ wide in the athwart-ship direction. Backscattered acoustic signals were received on a second linear receiving array that is oriented orthogonally to the transmit array to form a Mills cross (Mills and Little, 1953) that can be steered to form 256 beams over a $128^{\circ}$ sector of a plane. The receive beam width at broadside is nominally $0.5^{\circ}$ wide in the athwart-ship direction, resulting in an effective beam pattern (transmit and receive arrays combined) that is $1^{\circ} \times 0.5^{\circ}$. The native sampling scheme for the Reson 7125 produces pairs of 16 bit integers representing amplitude and phase for each sample in each beam. Each beam is sampled at a rate of approximately $34.5 \mathrm{ksamples} / \mathrm{s}$. The transmitted pulse length for the data used here is $300 \mu \mathrm{s}$. Data were collected at a ping rate that varied between 5 and 10 pings/s.

An example of the acoustic backscatter data collected from the multibeam is shown in Fig. 3. These data correspond to a "snapshot" in time of the bubble clouds: The

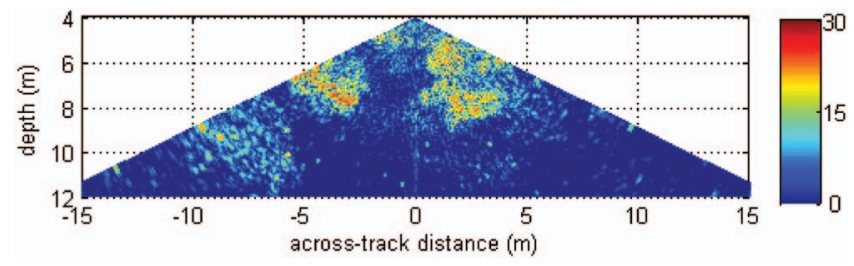

FIG. 3. (Color) An example of the multibeam data collected during the experiment. The data represent the combined 256 beams equally spaced over $\pm 64^{\circ}$. The color scale represents relative intensity in decibels.

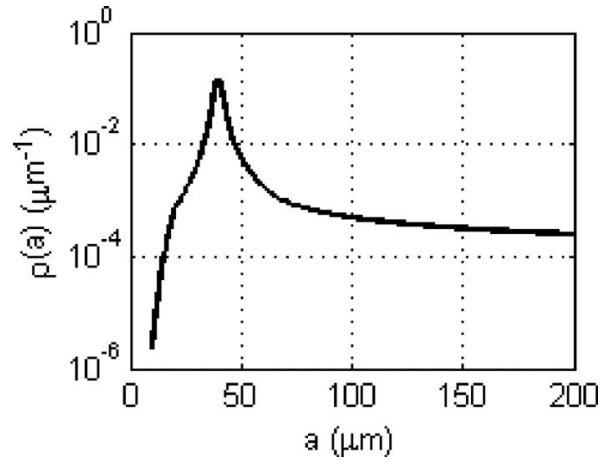

FIG. 4. The relative contributions of different bubble sizes at $400 \mathrm{kHz}$ for bubble distributed as shown in Fig. 2.

pulse travel times for the data shown in Fig. 3 range from 0 to $23 \mathrm{~ms}$. The regions corresponding to higher backscatter levels are areas in which there are bubbles. The radius of a resonant bubble at $400 \mathrm{kHz}$ at a depth of $6 \mathrm{~m}$ is approximately $10 \mu \mathrm{m}$ using the simplified expression for resonance frequency given by Clay and Medwin (1977, p. 197), which is at the small size limit for the bubble size distribution shown in Fig. 2. At such high frequencies, however, larger off-resonance bubbles can make an appreciable contribution to the backscatter. Dahl (2001) suggested a useful metric for evaluating the relative contribution of a distribution of bubble sizes to backscatter at a single frequency by defining the following density function $\rho\left(a_{s}\right)$ :

$$
\rho\left(a_{s}\right)=\frac{\sigma_{\mathrm{bs}}\left(a_{s}\right) n\left(a_{s}\right)}{\int \sigma_{\mathrm{bs}}(a) n(a) d a},
$$

where $a_{s}$ is the radius of the bubble contributing to the backscatter and $n$ is the bubble size distribution. The quantity $\sigma_{\mathrm{bs}}(a)$ is the backscattering cross section for a single bubble of radius $a$ (Clay and Medwin, 1977) as follows:

$$
\sigma_{\mathrm{bs}}(a)=\frac{a^{2}}{\left[\left(f_{0} / f\right)^{2}-1\right]^{2}+\delta^{2}},
$$

where $f_{0}$ is the resonance frequency of a bubble with radius $a, f$ is the frequency for the incident acoustic wave, and $\delta$ is the total damping coefficient accounting for viscous losses, thermal losses, and reradiation. Equation (9) is shown in Fig. 4 , and shows that the $400 \mathrm{kHz}$ measurement will be dominated by bubbles with radii near $40 \mu \mathrm{m}$, four times larger than the resonant bubble size, with bubbles whose size is between 35 and $45 \mu \mathrm{m}$ contributing to $68 \%$ of the overall scattering cross section.

It is important to note that one of the limitations of this experiment was that occasional dense bubble clouds would sweep directly underneath the multibeam transducers, rendering the measurements effectively useless. These instances are thought to represent very dense bubble clouds such as those that would be found directly under newly formed breaking waves (i.e., $\alpha$-plumes) Such instances were manually removed from the data that follow. Although this makes the multibeam measurements less than optimal, creating a potential bias in any of the results due to human intervention in the data, enough measurements (more than 7000 pings) 


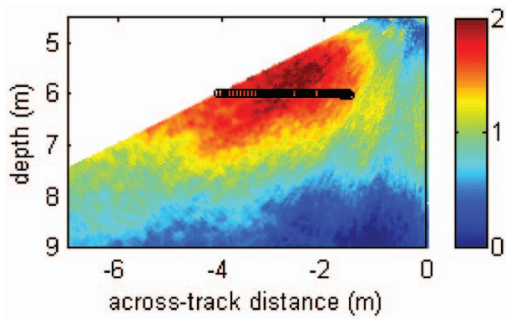

FIG. 5. (Color) The average backscattered intensity (proportional to the bubble number density) for the 365 pings (70 distinct bubble clouds) used in this work. The relative color scale is logarithmic. The black circles at a depth of $6 \mathrm{~m}$ show the individual sampling locations used to estimate the pair correlation function.

have been retained in order to make these data statistically significant of, at least, the less dense bubble clouds.

\section{Pair correlation function estimate}

One of the basic assumptions inherent in both Eq. (8) and in the treatment of propagation described by Weber et al. (2007) is that of statistical stationarity. Stationary data records can be difficult to achieve with transient forcing events such as breaking waves, where the bubble cloud appears at random locations within the multibeam field of view, and where the bubble number density is expected to change rapidly in time. For the data discussed here, this issue is exacerbated by the ship traveling through the water at $4 \mathrm{~m} / \mathrm{s}$ : Bubble clouds may be encountered at any stage of their evolution. To resolve this problem, the approach taken here is to examine multiple bubble clouds, identify both a center of mass and a measure of the void fraction for each cloud, and to then conduct the analysis described in Sec. II for clouds that have a similar location and void fraction. Cloud centers are estimated by calculating both a weighted mean beam number $\bar{b}$ and range $\bar{r}$ as follows:

$$
\begin{gathered}
\bar{b}=\frac{\sum_{i=1}^{N} A_{i}^{2} b_{i}}{\sum_{i=1}^{N} A_{i}^{2}}, \\
\bar{r}=\frac{\sum_{i=1}^{M} A_{i}^{2} r_{i}}{\sum_{i=1}^{M} A_{i}^{2}} .
\end{gathered}
$$

The weights are the backscattered intensity, a quantity that is proportional to the scattering cross section and therefore to the bubble number density (Clay and Medwin, 1977, p. 203). A proxy for void fraction is estimated by summing the backscattered pressure magnitude squared over a specified region. In this work, the interest will be in bubble clouds whose center is located at an across-track range of $-3.0 \pm 1.0 \mathrm{~m}$ on the port side, and the region over which the void fraction is calculated extends for approximately $3 \mathrm{~m}$ in all directions away from the cloud center. With this focus in bubble cloud location, and including only those pings that have void fractions within $\pm 25 \%$ of the average observed void fraction for clouds at this location, the resulting data set includes 365 pings over approximately 70 individual clouds. The average backscattered intensity for these pings is shown in Fig. 5.

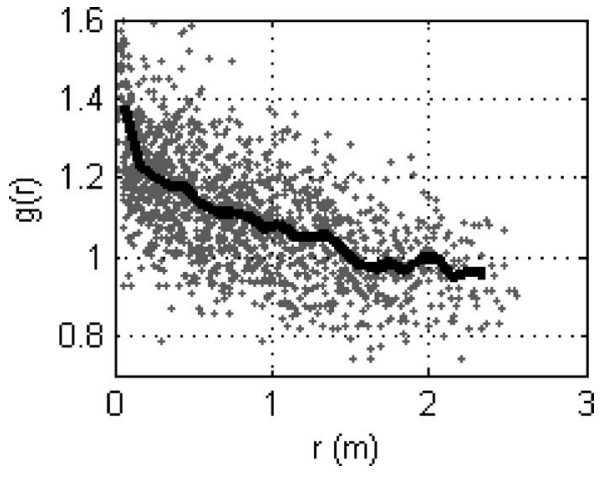

FIG. 6. Individual estimates of the pair correlation (gray dots) and the pair correlation function $g(r)$ plotted as a function of range $r$ (black line).

The pair correlation function was calculated for these data using Eq. (8) at measurement locations whose depth was $6 \mathrm{~m}$ and across-track ranges were within $1.5 \mathrm{~m}$ of the cloud center $(-3.0 \mathrm{~m})$, as shown in Fig. 5. The ensemble averages in Eq. (8) correspond to an average over the subset of 365 pings. The measurement locations shown in Fig. 5 correspond to 55 different beams, resulting in 1485 individual estimates of the pair correlation at different lengths. These estimates are binned at $10 \mathrm{~cm}$ increments, according to the separation distance for each pair, and bin averages are computed in order to find a smoothed pair correlation function. Both the individual estimates and the smoothed pair correlation function are shown in Fig. 6.

The estimate for the pair correlation function shown in Fig. 6 shows an approximately linear decay with increasing range, reaching a maximum value of 1.25 . The pair correlation function is greater than 1 at all ranges less than $1.5 \mathrm{~m}$, meaning that given the location of a single bubble, it is more likely to find another bubble less than $1.5 \mathrm{~m}$ away than it is to find one that is greater than $1.5 \mathrm{~m}$ away. At ranges larger than $1.5 \mathrm{~m}$, the pair correlation function is approximately equal to 1 , meaning that there is no statistical dependency between the locations of bubbles separated by distances larger than $1.5 \mathrm{~m}$. The pair correlation function shown in Fig. 6 appears to deviate from its linear behavior at the shortest ranges, less than $\sim 5 \mathrm{~cm}$. Pair correlation estimates at these short ranges are derived from adjacent beams, a situation that can introduce an anomalously high result due to overlapping beams. The beam pointing angles for the Reson 7125 are calculated such that their beam patterns will overlap at the half-power points $(-3 \mathrm{~dB})$. Thus, a significant portion of the sampling volume will be shared between adjacent beams, violating the assumption of unique bubble populations in the derivation of Eq. (8). The measurements corresponding to the shortest range $(5 \mathrm{~cm})$ are not used in the analysis that follows.

\section{THE EFFECT ON THE ACOUSTIC FIELD}

Weber et al. (2007) numerically integrated Eq. (1) in order to calculate the effect of bubble clustering on the attenuation of the average field for a specific geometry and bubble cloud size, finding that clustering can cause the attenuation to diverge from the nonclustered case above some 
bubble number density. Without a specific case study in mind (beyond the general case of bubble clouds under breaking waves), the approach here is to more generally analyze the effect of clustering on attenuation. This is done by first expanding Eq. (1) for the case of nonclustered bubbles to include the triple scattering term

$$
\begin{aligned}
\langle p(\vec{r})\rangle= & p_{0}(\vec{r})+S \int_{V} p_{0}\left(\vec{r}_{i}\right) G\left(\vec{r}, \vec{r}_{i}\right) n\left(\vec{r}_{i}\right) d \vec{r}_{i}+S^{2} \int_{V} \int_{V} p_{0}\left(\vec{r}_{i 1}\right) G\left(\vec{r}_{i 1}, \vec{r}_{i 2}\right) G\left(\vec{r}, \vec{r}_{i 2}\right) n\left(\vec{r}_{i 1}\right) n\left(\vec{r}_{i 2}\right) d \vec{r}_{i 1} d \vec{r}_{i 2} \\
& +S^{3} \int_{V} \int_{V} \int_{V} p_{0}\left(\vec{r}_{i 1}\right) G\left(\vec{r}_{i 1}, \vec{r}_{i 2}\right) G\left(\vec{r}_{i 2}, \vec{r}_{i 3}\right) G\left(\vec{r}, \vec{r}_{i 3}\right) n\left(\vec{r}_{i 1}\right) n\left(\vec{r}_{i 2}\right) n\left(\vec{r}_{i 3}\right) d \vec{r}_{i 1} d \vec{r}_{i 2} d \vec{r}_{i 3 i}+\cdots \\
= & p_{0}(\vec{r})+p_{1}(\vec{r})+p_{2}(\vec{r})+p_{3}(\vec{r})+\cdots,
\end{aligned}
$$

where the ellipsis indicates that this iterated multiple scattering series carries on ad infinitum. The term $S$ in Eq. (12) is a shorthand notation for the integral of the scattering coefficient $s$ weighted by the bubble size distribution $n(a)$ over all bubble sizes $a$, which is assumed here to be separable from the integrals over position. A shorthand notation of each scattering term has also been introduced, where $p_{1}$ represents the single scattered pressure, $p_{2}$ represents the double scattered pressure, and $p_{3}$ represents the tripled scattered pressure.

Equation (12) can be reduced by applying the Helmholtz operator $\left(\nabla^{2}+k_{0}^{2}\right)$ to both sides of the equation in a manner similar to that done by Foldy (1945) as follows:

$$
\begin{aligned}
\left(\nabla^{2}+k_{0}^{2}\right)\langle p(\vec{r})\rangle & =0-4 \pi S-4 \pi S^{2} \int_{V} p_{0}\left(\vec{r}_{i}\right) G\left(\vec{r}, \vec{r}_{i}\right) n\left(\vec{r}_{i}\right) \mathrm{d} \vec{r}_{i}-4 \pi S^{3} \int_{V} \int_{V} p_{0}\left(\vec{r}_{i 1}\right) G\left(\vec{r}_{i 1}, \vec{r}_{i 2}\right) G\left(\vec{r}, \vec{r}_{i 2}\right) n\left(\vec{r}_{i 1}\right) n\left(\vec{r}_{i 2}\right) d \vec{r}_{i 1} d \vec{r}_{i 2}-\cdots \\
& =-4 \pi S\left[p_{0}(\vec{r})+p_{1}(\vec{r})+p_{2}(\vec{r})+\cdots\right]=-4 \pi S\langle p(\vec{r})\rangle .
\end{aligned}
$$

Equation (13) is identical in result, and, essentially, method of derivation, to the result given by Foldy (1945), and can be solved to find an effective medium wave number $k$ as follows:

$$
k^{2}=k_{0}^{2}+4 \pi S=k_{0}^{2}+4 \pi \int s(a) n(a) d a .
$$

When clustering is present, Eq. (12) takes the form

$$
\begin{aligned}
\langle p(\vec{r})\rangle= & p_{0}(\vec{r})+S \int_{V} p_{0}\left(\vec{r}_{i}\right) G\left(\vec{r}, \vec{r}_{i}\right) n\left(\vec{r}_{i}\right) d \vec{r}_{i}+S^{2} \int_{V} \int_{V} p_{0}\left(\vec{r}_{i 1}\right) G\left(\vec{r}_{i 1}, \vec{r}_{i 2}\right) G\left(\vec{r}, \vec{r}_{i 2}\right) g\left(\left|\vec{r}_{i 1}-\vec{r}_{i 2}\right|\right) n\left(\vec{r}_{i 1}\right) n\left(\vec{r}_{i 2}\right) d \vec{r}_{i 1} d \vec{r}_{i 2} \\
& +S^{3} \int_{V} \int_{V} \int_{V} p_{0}\left(\vec{r}_{i 1}\right) G\left(\vec{r}_{i 1}, \vec{r}_{i 2}\right) G\left(\vec{r}_{i 2}, \vec{r}_{i 3}\right) G\left(\vec{r}, \vec{r}_{i 3}\right) g\left(\left|\vec{r}_{i 1}-\vec{r}_{i 2}\right|,\left|\vec{r}_{i 2}-\vec{r}_{i 3}\right|\right) n\left(\vec{r}_{i 1}\right) n\left(\vec{r}_{i 2}\right) n\left(\vec{r}_{i 3}\right) d \vec{r}_{i 1} d \vec{r}_{i 2} d \vec{r}_{i 3 i}+\cdots .
\end{aligned}
$$

The fourth term in Eq. (15) contains the triple correlation function $g\left(\left|\vec{r}_{i 1}-\vec{r}_{i 2}\right|,\left|\vec{r}_{i 2}-\vec{r}_{i 3}\right|\right)$ that is a function of the distances separating both the first two and the last two bubbles in a scattering chain. In some circumstances, this triple correlation function should be equivalent to the pair correlation function: This would occur, for example, when the joint probability density function (PDF) $n\left(\vec{r}_{i 1}, \vec{r}_{i 2}, \vec{r}_{i 3}\right)$ describing the locations of bubbles $i 1, i 2$, and $i 3$ is equal to the product of the joint pdf $n\left(\vec{r}_{i 1}, \vec{r}_{i 2}\right)$ and the marginal pdf $n\left(\vec{r}_{i 3}\right)$. This scenario could be expected to occur when $i 2$ and $i 3$ are far enough apart to be statistically independent (greater than $1.5 \mathrm{~m}$ for the bubble clouds examined in Sec. III). For bubble clouds in which the size of the bubble cloud, as well as the volume of integration for the integrals in Eq. (15), is large, the triple correlation function would be approximately equal to the double correlation function for the majority of the integration domain.

Equation (15) can also be reduced by applying the Helmholtz operator to each of the terms separately as follows: 


$$
\begin{aligned}
\left(\nabla^{2}+k_{0}^{2}\right)\langle p(\vec{r})\rangle= & 0-4 \pi S-4 \pi S^{2} \int_{V} p_{0}\left(\vec{r}_{i 1}\right) G\left(\vec{r}_{i 1}, \vec{r}\right) g\left(\left|\vec{r}_{i 1}-\vec{r}\right|\right) n\left(\vec{r}_{i 1}\right) d \vec{r}_{i 1}-4 \pi S^{3} \int_{V} \int_{V} p_{0}\left(\vec{r}_{i 1}\right) G\left(\vec{r}_{i 1}, \vec{r}_{i 2}\right) G\left(\vec{r}_{i 2}, \vec{r}\right) g\left(\mid \vec{r}_{i 1}\right. \\
& \left.-\vec{r}_{i 2}|,| \vec{r}_{i 2}-\vec{r} \mid\right) n\left(\vec{r}_{i 1}\right) n\left(\vec{r}_{i 2}\right) d \vec{r}_{i 1} d \vec{r}_{i 2}-\cdots .
\end{aligned}
$$

Unlike Eq. (13), the application of the Helmholtz operator does not result in the reduction by one of the scattering chain hierarchies. Instead, correction terms must be included as follows:

$$
\begin{aligned}
\left(\nabla^{2}+k_{0}^{2}\right)\langle p(\vec{r})\rangle= & -4 \pi S\left[p_{0}(\vec{r})+p_{1}(\vec{r})+p_{2}(\vec{r})+\cdots\right]-4 \pi S^{2} \int_{V} p_{0}\left(\vec{r}_{i 1}\right) G\left(\vec{r}_{i 1}, \vec{r}\right)\left[g\left(\left|\vec{r}_{i 1}-\vec{r}\right|\right)-1\right] n\left(\vec{r}_{i 1}\right) d \vec{r}_{i 1} \\
& -4 \pi S^{3} \int_{V} \int_{V} p_{0}\left(\vec{r}_{i 1}\right) G\left(\vec{r}_{i 1}, \vec{r}_{i 2}\right) G\left(\vec{r}_{i 2}, \vec{r}\right)\left[g\left(\left|\vec{r}_{i 1}-\vec{r}_{i 2}\right|,\left|\vec{r}_{i 2}-\vec{r}\right|\right)-g\left(\left|\vec{r}_{i 1}-\vec{r}\right|\right)\right] n\left(\vec{r}_{i 1}\right) n\left(\vec{r}_{i 2}\right) d \vec{r}_{i 1} d \vec{r}_{i 2}-\cdots .
\end{aligned}
$$

The third term in Eq. (17), which is based on the difference between the triple and pair correlation functions, should vanish when the volume of integration is much larger than the length over which bubble positions are found to be statistically dependent. This is essentially the same argument made when using the quasicrystalline approximation (QCA) to account for the hole correction in very densely packed scatterers (Lax, 1952). When this occurs, Eq. (17) can be approximated as

$$
\begin{aligned}
\left(\nabla^{2}+k_{0}^{2}\right)\langle p(\vec{r})\rangle \cong & -4 \pi S\left[p_{0}(\vec{r})+p_{1}(\vec{r})+p_{2}(\vec{r})+\cdots\right]-4 \pi S^{2} \\
& \times \int_{V} p_{0}\left(\vec{r}_{i 1}\right) G\left(\vec{r}_{i 1}, \vec{r}\right)\left[g\left(\left|\vec{r}_{i 1}-\vec{r}\right|\right)-1\right] \\
& \times n\left(\vec{r}_{i 1}\right) d \vec{r}_{i 1},
\end{aligned}
$$

leading to a new effective wave number that accounts for clustering as follows:

$$
\begin{aligned}
k^{2} \cong & k_{0}^{2}+4 \pi S+4 \pi S^{2}\langle p(\vec{r})\rangle^{-1} \int_{V} p_{0}\left(\vec{r}_{i 1}\right) \\
& \times G\left(\vec{r}_{i 1}, \vec{r}\right)\left[g\left(\left|\vec{r}_{i 1}-\vec{r}\right|\right)-1\right] n\left(\vec{r}_{i 1}\right) d \vec{r}_{i 1} .
\end{aligned}
$$

There are several features of Eq. (19) that are worth pointing out. First, as the clustering grows weaker and the pair correlation function becomes close to 1 , this solution converges to the nonclustered solution given in Eq. (14). Second, the correction for clustering grows both as the volume of bubbles grows and as the distance of propagation grows. For example, consider a plane wave that would propagate in the bubble-free medium as $\exp \left(-j k_{0} x\right)$. The effective wave number described by Eq. (19) would then be written as

$$
\begin{aligned}
k^{2} \cong & k_{0}^{2}+4 \pi S+4 \pi S^{2} \\
& \times \frac{\int_{V} \exp \left(-j k_{0} x_{i 1}\right) G\left(\vec{r}_{i 1}, \vec{r}\right)\left[g\left(\left|\vec{r}_{i 1}-\vec{r}\right|\right)-1\right] n\left(\vec{r}_{i 1}\right) d \vec{r}_{i 1}}{\exp (-j k x)} .
\end{aligned}
$$

Considering a widely separated source and receiver with a bubble cloud in between, the correction term in Eq. (20) will grow as either the size and or density of the bubble cloud grows, thereby decreasing the magnitude of the term in the denominator. This is in qualitative agreement with result found by Weber et al. (2007), who found that the relative importance of double scattering increases with both the number of bubbles and the bubble cloud size.

Evaluating Eq. (20) is situational dependent, not only on the bubble cloud parameters but also on the source/receiver geometry. However, a simple example is provided here in which Eq. (20) is numerically evaluated. The effective wave number $k$ is solved for iteratively because of its presence in both the left-hand side and in the denominator of the third term on the right-hand side of Eq. (20). Starting with an initial guess for the effective wave number $k_{g}$, which is used in the denominator on the right-hand side, Eq. (20) is evaluated in order to find an estimate for $k$. This new estimate is then used as the new $k_{g}$ until the magnitude of the terms $\exp \left(-j k_{g} x\right)$ and $\exp (-j k x)$ are within $1 \%$ of each other. In this manner, Eq. (20) has been evaluated using the pair correlation function shown in Fig. 6, the bubble size distribution shown in Fig. 2, and assuming that the hydrophone [i.e., the field point $\vec{r}$ in Eq. (20)] observes incident plane waves at a position that is located $1 \mathrm{~m}$ from the edge of a bubble cloud such that the term in the denominator of Eq. (20) includes the attenuation $\exp (-\operatorname{Im}\{k\} 1)$. The bubble cloud is assumed to be large so that the approximation (analogous to the QCA) leading to Eq. (20) is valid. The results shown in Fig. 7 describe a difference in attenuation between the clustered and nonclustered bubble clouds that varies between $20 \%$ and $80 \%$ over a frequency range between 10 and $350 \mathrm{kHz}$. The attenuation for the clustered bubble cloud is lower than that predicted for the nonclustered bubble cloud at frequencies between 10 and $230 \mathrm{kHz}$, and higher at frequencies between 230 and $350 \mathrm{kHz}$.

The reason for the transition from a lower than expected attenuation to a higher than expected attenuation in Fig. 7 is based on the competition between the last two terms in Eq. (20). Considering that the last two terms in Eq. (20) are small in comparison to $k_{0}^{2}$, the attenuation can be written as 


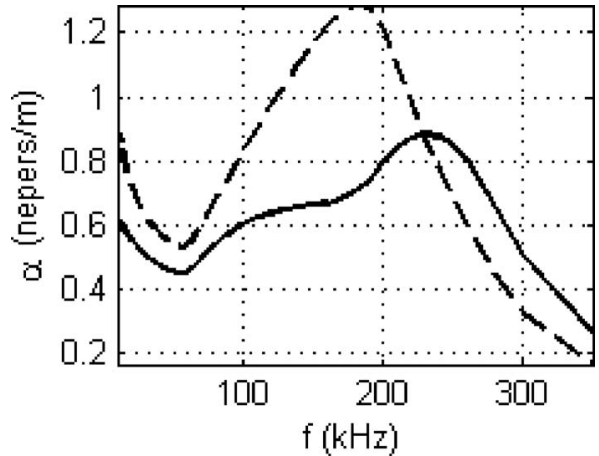

FIG. 7. The predicted attenuation for nonclustered [Eq. (14); dashed line] and clustered bubble clouds [Eq. (18), solid line].

$$
\begin{aligned}
\alpha \cong & -\frac{2 \pi}{k_{0}} \operatorname{Im}\left\{S+S^{2}\right. \\
& \left.\times \frac{\int_{V} \exp \left(-j k_{0} x_{i 1}\right) G\left(\vec{r}_{i 1}, \vec{r}\right)\left[g\left(\left|\vec{r}_{i 1}-\vec{r}\right|\right)-1\right] n\left(\vec{r}_{i 1}\right) d \vec{r}_{i 1}}{\exp (-j k x)}\right\} .
\end{aligned}
$$

From Fig. 6, the pair correlation function can be approximated as $g=1.25-(0.25 / 1.25) r$, in which case the volume integral in Eq. (21) is proportional to $1 / j k_{0}$ and the second term in the brackets of Eq. (21) is proportional to $S^{2} / j k_{0}$. The imaginary part of this latter quantity, $\operatorname{Im}\left\{S^{2} / j k_{0}\right\}$, has been plotted as a function of frequency in Fig. 8. Noting that $\operatorname{Im}\{S\}$ is negative at all frequencies (hence the positive attenuation in Fig. 7), clustering should cause the attenuation to decrease where $\operatorname{Im}\left\{S^{2} / j k_{0}\right\}$ is greater than zero and increase where $\operatorname{Im}\left\{S^{2} / j k_{0}\right\}$ is less than zero. A comparison of Figs. 7 and 8 shows that this is occurring here: The attenuation for the clustered bubble cloud becomes higher than that for the nonclustered bubble cloud at $230 \mathrm{kHz}$, where the quantity $\operatorname{Im}\left\{S^{2} / j k_{0}\right\}$ changes sign.

\section{DISCUSSION}

When using stochastic approaches for examining the effect of bubbles on the propagation of acoustic waves, it is often assumed that the positions of the bubbles are statistically independent random variables. This assumption has been tested here using multibeam sonar backscatter from bubbles under oceanic breaking waves in an open ocean condition at a wind speed of $14.6 \mathrm{~m} / \mathrm{s}$, and found to be not

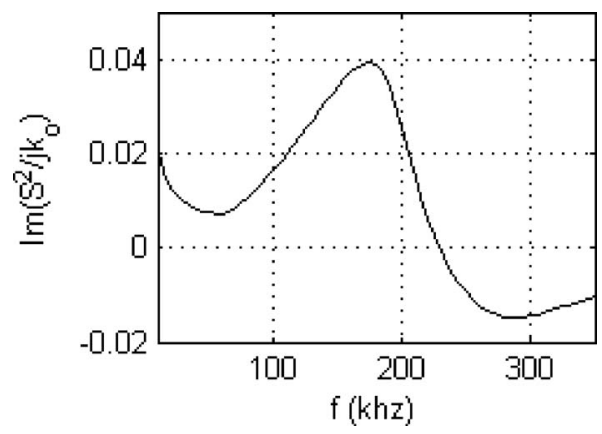

FIG. 8. A term proportional to the clustering induced modification of the attenuation plotted as a function of frequency. generally valid. The multibeam data indicate that the bubble positions were positively correlated (clustered) at ranges less than $1.5 \mathrm{~m}$, and independent of one another at greater ranges. By itself, this finding does not mean that commonly used effective medium theories are in error. For scenarios where only single scattering is non-negligible, the important scattering paths contain an interaction with only one bubble and so the average of the scattered waves associated with these paths depends only on the marginal probability density function describing the location of individual bubbles. This might happen at lower wind speeds where the bubble number density is less. The correlations between the fluctuating positions of bubbles become important for the mean acoustic field when double scattering terms (or higher) become important.

A correction term for the average acoustic field that accounts for bubble clustering has been suggested in this paper. This correction term vanishes when the bubble positions are statistically independent (i.e., when the pair correlation function $g$ is equal to 1), and, because it is based on a double scattering term in an iterated multiple scattering series, will become negligible when double scattering is negligible. The correction term used in this work is an approximation similar to the QCA described by Lax (1952). This approximation suggests that the clustering correction for double scattering dominates corrections for higher orders of scattering, and is expected to hold true when the positions of the bubbles are statistically dependent only on ranges much shorter than the size of the bubble cloud contributing to the mean acoustic field.

Using the pair correlation function estimated from the multibeam data, and a predicted bubble size distribution for oceanic breaking waves, it was found that the application of the correction term caused a substantial deviation in the mean acoustic field from that which would be predicted in the absence of clustering. Whether or not the clustered bubble cloud attenuation is higher or lower than the nonclustered bubble cloud attenuation will be a function of the frequency dependence of the bubble scattering coefficient integrated over the bubble size distribution. This means that the correction term will be a function of both the statistical dependency between the positions of the bubbles and the size distribution of the bubbles. It is important to note that the data described here, and their relationship to the importance of bubble clustering, can be considered only an example of the type of fluctuating spatial structures that exist within bubble clouds in the ambient ocean. It is quite likely that scenarios exist where the bubble clustering (and associated correlation functions) are either stronger or weaker, and presumably there is a relationship between the observed correlations and the bubble cloud forcing functions (e.g., different wind speeds may mean different correlation functions).

The deviations in the mean acoustic field were found to exist over a wide frequency range, from 10 to $350 \mathrm{kHz}$. However, the implicit assumptions in this analysis were that the clustering observed with the $400 \mathrm{kHz}$ multibeam was the same across all bubble sizes, and that the overall bubble size distribution was adequately described by a model of this distribution for a $\beta$-plume. In the absence of a multifrequency multibeam sonar and direct measurements of the bubble size 
distribution, these assumptions make the problem tractable. There is a possibility that these assumptions may not have been appropriate. For example, the assumption that all bubbles respond to the fluid flow in the same way and exhibit identical clustering may not hold true for all bubble sizes. A separate body of research describing the clustering of particles in turbulent flow fields (e.g., Eaton and Fessler, 1994) suggests that not all bubbles will respond to the flow fields generating the clustering equally. This research indicates that the degree to which bubble size sorting occurs in the ocean should depend on the Stokes number (defined as the ratio of the bubble response time to a characteristic time scale of the flow): Bubbles with a Stokes number near unity should become clustered. Because the response time of a bubble, which can be thought of as the time required for a bubble released from rest into a fluid flow to reach a steady velocity, is size dependent it is possible that naturally occurring bubble distributions created in dynamic flows are being spatially sorted by turbulent flows. If this were occurring, the pair correlation would be a function of both separation distance and bubble size. Because the single frequency multibeam observations used in this paper were unable to distinguish between different bubble sizes, the potential for bubble size sorting has been neglected.

This work has been focused on understanding the effects of clustering on short-range acoustic propagation. Clustering may also play an important role in long-range propagation scenarios, particularly in shallow water environments where there may be multiple surface bounces and interactions with near-surface bubble clouds. In these types of scenarios, clustering may be observed in two different regimes. The first is what has been analyzed here: clustering within individual bubble clouds, at scales that are less than the bubble cloud sizes. Clustering could also be used to describe the spacing of the bubble clouds themselves. In this intercloud spacing regime, a pair correlation function would be greater than 1 (i.e., showing statistical dependence on the positions of bubbles) at length scales up to the nominal size of a bubble cloud. This would occur even if the intracloud clustering was not present; it can be viewed as a statement that if there is a bubble at a given location it is likely that the bubble is inside of a bubble cloud. It would then follow that at ranges greater than the nominal size of a bubble cloud, the pair correlation function would be less than 1 . That is, given the location of a single bubble, it is less likely to find a second bubble outside of the cloud. Note that the likelihood at large ranges is not zero: A second bubble may be part of the background bubble population or a second cloud. Given the form of this intercloud pair correlation function, it would be possible to analyze the effect on the acoustic field using the same framework established here. In addition to the change in correlation function, the main change would be the replacement of the free-space Green's function with a Green's function more appropriate for the case at hand (e.g., a shallow water waveguide). Thus, it is possible that at long ranges, clustering will have analogous effects to those analyzed here at short ranges.

\section{ACKNOWLEDGMENTS}

This work was supported by the Office of Naval Research Ocean Acoustics program (Code 321). The methodology for using high frequency multibeam measurements to estimate pair correlation functions was developed as part of the author's Ph.D. dissertation while under the guidance of Anthony P. Lyons and David L. Bradley at the Applied Research Laboratory at Penn State. Both Lyons and Bradley also provided numerous helpful suggestions in the preparation of this manuscript, for which the author is very grateful. The author would also like to express his gratitude to Nicholas Makris at the Massachusetts Institute of Technology for extending the invitation to join the cruise on which these data were collected, to Chris Malzone and Reson for making the multibeam sonar available, and, last but not the least, to the Captain, crew, and scientific party onboard the R/V Hugh R. Sharp, all of whom weathered a rather uncomfortable storm while these data were being collected.

Benoit-Bird, K., and Au, W. (2003). "Hawaiian spinner dolphins aggregate midwater food resources through cooperative foraging," J. Acoust. Soc. Am. 114, 2300

Clay, C., and Medwin, H. (1977). Acoustical Oceanography: Principles and Applications (Wiley, New York).

Commander, K. W., and Prosperetti, A. (1989). "Linear pressure waves in bubbly liquids: Comparison between theory and experiments," J. Acoust. Soc. Am. 19, 732-746.

Crawford, G., and Farmer, D. (1987). "On the spatial distributions of ocean bubbles," J. Geophys. Res. 92, 8231-8243.

Dahl, P. (2001). "Bubble clouds and their transport within the surf zone as measured with a distributed array of upward-looking sonars," J. Acoust. Soc. Am. 109, 133-142.

Eaton, J., and Fessler, J. (1994). "Preferential concentration of particles by turbulence," Int. J. Multiphase Flow 20, 169-209.

Foldy, L. L. (1945). "The multiple scattering of waves, I. General theory of isotropic scattering by randomly distributed scatterers," Phys. Rev. 67, 107-119.

Gerlotto, F., and Paramo, J. (2003), “The three-dimensional morphology and internal structure of clupeid schools as observed using vertical scanning multibeam sonar," Aquatic Living Resources 16, 113-122.

Henyey, F. (1999). "Corrections to Foldy's effective medium theory for propagation in bubble clouds and other collections of very small scatterers," J. Acoust. Soc. Am. 105, 2149-2154.

Jones, C. D. (2003). "Water-column measurements of hydrothermal vent flow and particulate concentration using multibeam sonar," J. Acoust. Soc. Am. 114, 2300-2301.

Lax, M. (1952). "Multiple scatter of waves. II. The effective field in dense systems," Phys. Rev. 85, 621-629.

Mayer, L., Yanchao, L., and Melvin, G. (2002). "3D visualization for pelagic fisheries research and assessment," ICES J. Mar. Sci. 59, 216-255.

Mallock, A. (1910). "The damping of sound by frothy liquids," Proc. R. Soc. London, Ser. A 84, 391-395.

Medwin, H., and Clay, C. (1998), Fundamentals of Acoustical Oceanography, Academic, San Diego, CA.

Mills, B. Y., and Little, A. G. (1953). "A high resolution aerial system of a new type," Aust. J. Phys. 6, 272-278.

Monahan, E., and Lu, M. (1990). "Acoustically relevant bubble assemblages and their dependence on meteorological parameters," IEEE J. Ocean. Eng. 15, 340-349.

National Defense Research Committee (1946). Physics of Sound in the Sea, Division 6 Summary Technical Report (National Defense Research Committee, Washington, D.C.), Vol. 8.

Novarini, J., Keiffer, R., and Norton, G. (1998). "A model for variations in the range and depth dependence of the sound speed and attenuation induced by bubble clouds under wind-drive seas," IEEE J. Ocean. Eng. 23, 423-438.

Shaw, R., Kostinski, A., and Larsen, M. (2002). "Towards quantifying droplet clustering in clouds," Q. J. R. Meteorol. Soc. 128, 1043-1057.

Terrill, E., and Melville, W. (1997). "Sound speed measurements in the 
surface-wave layer," J. Acoust. Soc. Am. 102, 607-625.

Thorpe, S. (1982). "On the clouds of bubbles formed by breaking windwaves in deep water and their role in air-sea gas transfer," Philos. Trans. R. Soc. London, Ser. A 304, 155-210.

Trevorrow, M. (2005). "Volumetric multibeam sonar measurements of fish, zooplankton, and turbulence," Proceedings of the International Conference on Underwater Acoustic Measurements: Technologies \& Results, Crete, Greece, 28 June-1 July 2005.

Van Wijngaarder, L. (1972). "One-dimensional flow of liquids containing small gas bubbles," Annu. Rev. Fluid Mech. 2, 369-396.

Weber, T. C., Lyons, A. P., and Bradley, D. L. (2005). "An estimate of the gas transfer rate from oceanic bubbles derived from multibeam sonar observations of a ship wake," J. Geophys. Res. 110, C04005.

Weber, T. C., Lyons, A. P., and Bradley, D. L. (2007). "Acoustic propagation through clustered bubble clouds," IEEE J. Ocean. Eng. 32, 513-523.

Wood, A. B. (1930). A Textbook of Sound (MacMillan, New York).

Wu, J. (1981). "Bubble populations and spectra in near-surface ocean: Summary and review of field measurements," J. Geophys. Res. 86, 457-463.

Wu, J. (1994). "Bubbles in the near-surface ocean: Their various structures," J. Phys. Oceanogr. 24, 1955-1965.

Wu, J. (1998). "Bubbles in the near surface ocean: A general description," J. Geophys. Res. 93, 587-690. 